\title{
Strain-Specific Transcription and Translation of the BamHI Z Area of Epstein-Barr Virus
}

\author{
RUDOLF SEIBL, MANFRED MOTZ, AND HANS WOLF* \\ Max von Pettenkofer-Institut, University of Munich, 8000 Munich 2, Federal Republic of Germany
}

Received 27 March 1986/Accepted 21 August 1986

\begin{abstract}
The expression of the 1,800-base-pair BamHI $Z$ region of Epstein-Barr virus DNA was analyzed by hybrid-selected translation with several DNA subclones and RNA from different cell lines. Furthermore, large segments of the three reading frames extending in this area were expressed as fusion proteins into Escherichia coli. The fusion proteins were partially purified and used to immunize rabbits. These sera were used to confirm our mapping assignments and to identify the respective posttranslationally modified proteins in in vivo labeling experiments. The reading frame BRLF1 (the first reading frame starting in the BamHI R fragment in leftward orientation) encoded a 93- to 96-kilodalton (KDa) protein depending on the cell line. The molecular weight of in vivo-labeled proteins was increased relative to that of in vitro-translated proteins, indicating that a posttranslational modification had occurred. The BZLF1 reading frame encoded a 35-kDa protein. It was posttranslationally cleaved from a 38-kDa precursor in induced B95-8 and induced Raji cells and from a 40-kDa precursor in induced P3HR1 cells. In Raji cells superinfected with virus derived from P3HR1 cells, the protein seemed to be expressed both from endogeneous Raji genomes and from infecting genomes. The transcripts for the 93- to 96-kDa and the 35-kDa protein overlapped partially. The serum against the expressed third reading frame BZLF2 specifically precipitated a 140-kDa protein. This reading frame contains only 650 nucleotides, and therefore further coding sequences were presumably spliced to BZLF2. The latter is deleted in the Raji cell line; therefore, the observed 140kDa protein in superinfected Raji cells was expressed from infecting P3HR1 genomes.
\end{abstract}

Epstein-Barr virus (EBV), a member of the herpesvirus family, undergoes limited replication, presumably lifelong, in the oropharynxes of most individuals following primary infection $(16,28,34)$. Viral DNA can also be demonstrated lifelong in peripheral B lymphocytes of all seropositive individuals. These latently infected lymphocytes acquire the capacity for unlimited growth. In vitro, only B lymphocytes can be infected. Cell lines established from such experiments, from naturally infected peripheral lymphocytes, or from Burkitt lymphoma biopsies, are the only EBV-positive cell culture systems available. In these permanently growing cell lines, the virus remains essentially latent and only a limited set of viral genes is expressed. In some cell lines (e.g., B95-8, P3HR1), a low percentage of the cells constantly enter the productive cycle, and proteins from the early and late stages of virus replication and infectious virus are produced. The number of cells entering the lytic cycle is dramatically increased after induction with various agents, particularly with phorbol-12-mystrate-13-acetate (35) and butyric acid (10). Some nonproducer cell lines, like Raji, can be stimulated as well by these inducers to express viral proteins of the lytic cycle. The Raji cells are exceptional in that they produce only proteins of the early class. P3HR1 cells have two peculiarities: first, the produced virus is incapable of transforming cells and, second, the virus preparations contain a population of defective viruses. These defective particles contain four EBV DNA segments that are partially inverted and fused $(3,14,26)$. The defective DNA (het-DNA) is correlated with the capacity to induce the lytic cycle from latent genomes, e.g., after the superinfection of Raji cells $(14,24)$. The het-DNA BamHI $\mathrm{Z}$ area is fused in the opposite orientation to sequences from the BamHI W fragment $(3,6,26)$. Transfection of a cloned subfragment of

\footnotetext{
* Corresponding author.
}

the het-DNA containing the fused $\operatorname{BamHI} \mathrm{W}$ and $\mathrm{Z}$ fragments into latent hybrid D98/HR-1 cells (6) or of a standard BamHI Z fragment into Raji cells (30) trans-activates the latent EBV genome, presumably by proteins expressed from the transfected DNA.

By using hybrid-selected translation with RNA from induced P3HR1 cells, we (27) and others (5) mapped several proteins to the Bam $\mathrm{HI} \mathrm{Z}$ fragment. In further experiments, we used RNA from induced Raji cells and could map to this fragment proteins similar to that from P3HR1 cells but with a slightly differing molecular weight (manuscript in preparation). It should be noted that the proteins identified by this method are primary translation products (owing to the absence of posttranslational modifications in the in vitro translation system). Monospecific sera or monoclonal antibodies are therefore necessary to positively identify which of the in vivo-labeled EBV proteins examined in several laboratories in terms of time-dependent synthesis, modification, correlation to antigenic groups, and sensitivity to inhibitors are encoded by the various genes.

In this paper, we describe the detailed examination of the cell-line-specific expression of the BamHI Z reading frames. By using hybrid-selected translation with several subclones of this region and RNA from different cell lines, we could correlate our previously mapped in vitro-translated proteins to two of the reading frames and obtain basic information about the transcriptional organization. The protein encoded by the third reading frame, which is deleted in EBV-DNA of the Raji strain (22), was not detectable by in vitro translation. We further expressed large parts of the three reading frames in Escherichia coli and immunized rabbits with the partially purified proteins. These rabbit sera were used in immunoprecipitation reactions to assign the mapped proteins to the single reading frames and to identify the respective posttranslationally modified proteins in in vivo labeling 


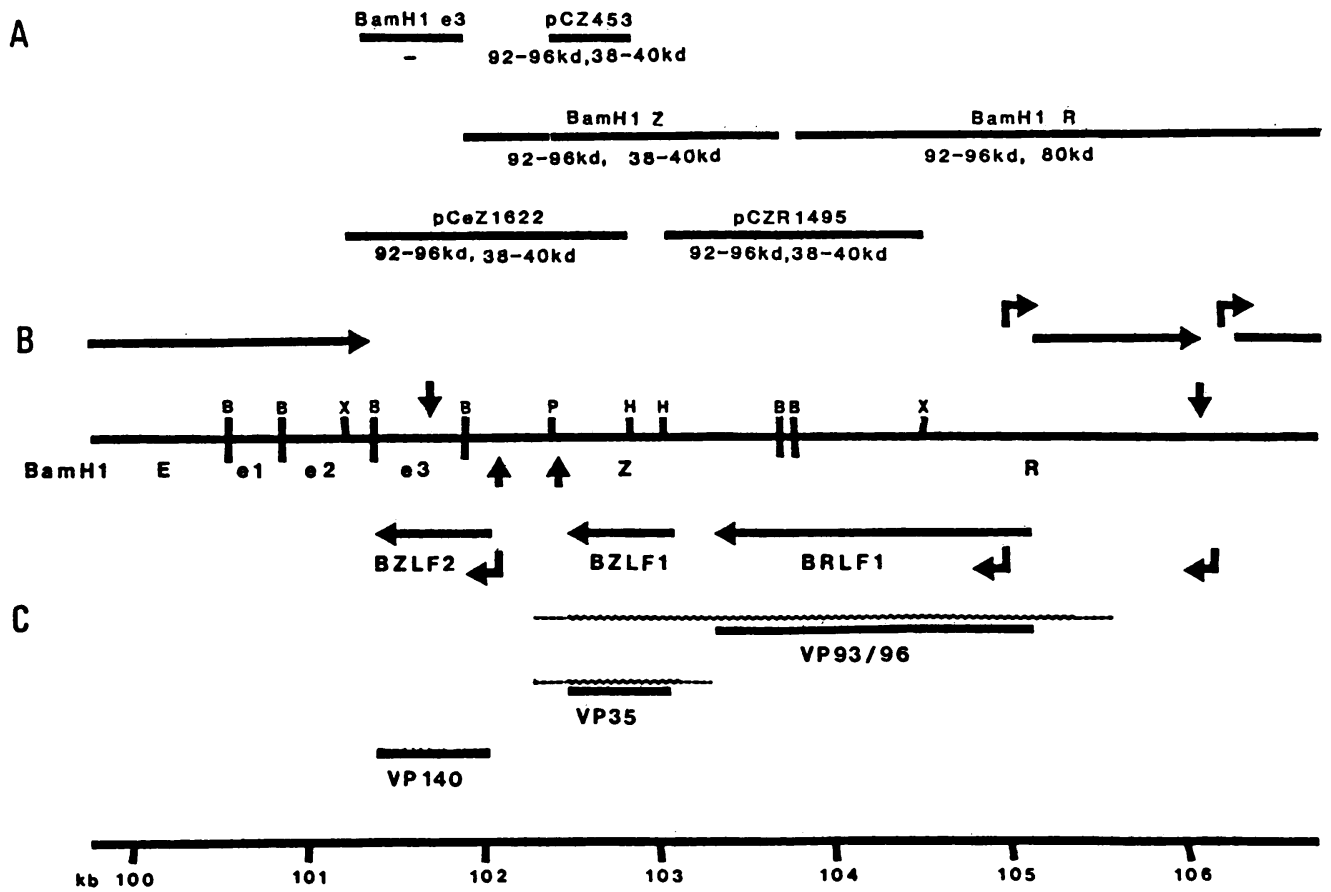

FIG. 1. Map of the BamHI Z area with a summary of the data. (A) DNA fragments used in the experiments. The figure gives the plasmids used for hybrid-selected translation, the proteins mapped to them, and the fragments of the EBV genome used for protein expression. The BamHI e3 fragment was cloned in a pUR expression vector. This plasmid was simultaneously used for hybrid-selected translation and for the expression of parts of the BZLF2 reading frame. Fragment Z453 is contained in a pUC vector (pCZ453) and was used in this form for hybrid selection. The same fragment was also cloned into a pUR plasmid (pRZ453) for the expression of parts of the BZLF1 reading frame. The BamHI Z and R fragments cloned in pBR322 (28) and the plasmid pCeZ1622 were used only for hybrid-selected translation. Fragment ZR1495 was cloned in pUC19 (pCZR1495) and used for hybrid-selected translation; it was also cloned in a pUR vector (pRZR1495) for the expression of parts of BRLF1 reading frame. Fragments eZ1622 and ZR1495 were also cloned in M13, and the single-stranded phage DNA of clones pPeZ1622 and pPZR1495 was used for hybrid-selected translation (see Materials and Methods). (B) Organization of the BamHI Z region.

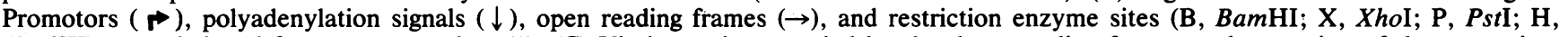
HindIII) were deduced from sequence data (1). (C) Viral proteins encoded by the three reading frames and extension of the transcripts according to the data obtained by hybrid-selected translation. The ends are punctured to show that the exact start and end are not defined.

experiments. The protein encoded by the third reading frame was also identified by immunoprecipitation with the appropriate serum.

\section{MATERIALS AND METHODS}

RNA manipulations. Tissue culture, RNA preparation, hybrid selection, and in vitro translation were done as described previously (27). Briefly, the cells were lysed 2 days after induction (with 40ng of phorbol-12-mystrate-13acetate per $\mathrm{ml}$ plus $3 \mathrm{mM}$ sodium butyrate) with $4 \mathrm{M}$ guanidine isothiocyanate-0.5 M 2-mercaptoethanol. The RNA was isolated by sedimentation through a $\mathrm{CsCl}$ cushion $\left(1.8 \mathrm{~g} / \mathrm{cm}^{3}\right)$. For hybrid selection, the DNA was spotted on small nitrocellulose filters and hybridized against the total RNA. Bound RNA was eluted by boiling. The selected mRNA was translated in vitro with a rabbit reticulocyte lysate.

Cloning of hybridization probes. The DNA sequence of the B95-8 strain (1) was analyzed with computer programs (7) for restriction enzyme sites and reading frames. The DNA was digested with the appropriate restriction enzymes (Boehringer Mannheim GmbH, Mannheim, Federal Republic of Germany) and run on agarose gels. Bands containing the desired sequences were excised, eluted electrophoretically, purified and concentrated with Elutip-d columns (Schleicher
\& Schuell, GmbH, Dassel, Federal Republic of Germany), ligated with T4 DNA ligase (Boehringer), and used to transform bacteria as described by Hanahan (9).

The Sall C fragment of EBV strain M-ABA DNA cloned in pHC79 (23) was digested with HindIII and XhoI. A 1,495-base-pair (bp) fragment containing parts of the BamHI $\mathrm{Z}$ and $\mathrm{R}$ fragments was ligated with HindIII- and SalIdigested pUC19 (19) to generate the plasmid pCZR1495 (Fig. 1). A 1,622-bp fragment covering the BamHI e3 fragment and parts of the BamHI e2 and Z fragments was cloned similarly to generate the plasmid pCeZ1622. The latter was digested with HindIII and PstI, and 453 bp of the BamHI Z fragment were cloned in pUC19 as pCZ453.

The inserts of pCeZ1622 and pCZR1495 were cloned in addition in M13mp8 (13). The single-stranded DNA of the bacteriophage particles from the resulting M13 clones pPeZ1622 and pPZR1495 resembles the opposite strands because the fragments in the two clones are in opposite orientation with respect to their restriction enzyme sites at the ends.

Cloning for expression of EBV proteins as bacterial fusion proteins. The theoretical secondary structure of the EBV proteins superimposed by their hydrophilicity and hydrophobicity was calculated with a computer program written in our laboratory following suggestions by $\mathrm{E}$. Golub and G. Cohen (4). Potential antigenic sites which are preferentially 
in hydrophilic $\beta$-turns were included in the expressed portions of the EBV proteins (for a detailed description, see reference 17).

The EBV proteins were expressed as fusion proteins with $\beta$-galactosidase in pUR plasmids (25). The viral reading frames were inserted in frame near the $C$ terminus of the $\beta$-galactosidase reading frame. The $B a m H I$ e 3 fragment was excised from pCeZ1622 and ligated with pUR278. In the resulting plasmid pRe3-521 (BamHI e3), which was also used for hybrid selection, the C-terminal 165 amino acids of the 670-bp BZLF2 frame were expressed. The end of the BZLF2 frame is within the $B a m H I$ e 3 fragment, and therefore the fusion protein ends with the EBV sequence. For the expression of the BRLF1 reading frame, the EBV-derived sequence of pCZR1495 was excised with HindIII and $X b a I$ and ligated with pUR288. In the resulting plasmid pRZR1495, again the $3^{\prime}$ part of the BRLF1 reading frame is fused in frame to the $\beta$-galactosidase reading frame with a few nucleotides from the pUC vector in between. The last 400 amino acids of the 1,800-bp BRLF1 frame were expressed with the EBV-encoded part again at the end of the fusion protein.

The reading frame BZLF1 contains one probably preferential antigenic site between amino acids 150 and 175. The expression of the appropriate sequences in a pUR vector was only possible after insertion of the EBV-derived sequences of pCZ453 after a HindIII-XbaI digest in PINIII B1 (11). From there, the EBV sequences were excised with $B a m H I$ and $X b a I$ and ligated with pUR289. In the resulting plasmid pRZ453, the last 330 nucleotides of the 600 -bp BZLF1 reading frame were fused to the $\beta$-galactosidase reading frame with a few nucleotides of PINIII B1 in between.

Expression and purification of the fusion proteins. The expression plasmids were transformed in E. coli JM103 (12). This strain represses the transcription of the fusion reading frame by overproduction of the lac repressor. Transcription was induced with $1 \mathrm{mM}$ isopropyl- $\beta-D-$ thiogalactopyranoside after the bacteria had grown to an optical density of 0.9 . The bacteria were grown for another 2 to $4 \mathrm{~h}$, sedimented, digested with lysozyme, and sonicated. After addition of $3 \%$ Triton X-100, the bacterial lysate was sedimented. Most of the bacterial proteins were dissolved in the supernatant. The expressed fusion proteins were found predominantly in the insoluble pellet. The pellet was dissolved in $8 \mathrm{M}$ urea-20 mM Tris ( $\mathrm{pH} \mathrm{8.0)-0.5 \%} \mathrm{mercaptoethanol} \mathrm{and} \mathrm{cen-}$ trifuged again. The supernatant with the dissolved fusion proteins was subsequently dialyzed against 6,4 , and $3 \mathrm{M}$ urea. Two bacterial proteins precipitated and could be separated by sedimentation. The $3 \mathrm{M}$ urea supernatant was dialyzed against phosphate-buffered saline, which led to the precipitation of a high portion of the fusion proteins as determined by a comparison to the supernatant. The precipitated fusion proteins could be isolated by sedimentation and were used for immunizing the rabbits.

Immunoprecipitation, polyacrylamide gel electrophoresis, and Western blotting. Raji cells were superinfected with virus isolated from P3HR1 cells as described previously (2). At $12 \mathrm{~h}$ postinfection, the cells were labeled for $4 \mathrm{~h}$ with 50 $\mu \mathrm{Ci}$ of $\left[{ }^{35} \mathrm{~S}\right]$ methionine per $\mathrm{ml}$. The induced cell lines were labeled 2 days after induction for $16 \mathrm{~h}$ with $20 \mu \mathrm{Ci}$ of $\left[{ }^{35} \mathrm{~S}\right]$ methionine per $\mathrm{ml}$. The cells were subsequently washed and lysed in immunoprecipitation buffer (1\% Triton X-100, $0.1 \%$ sodium dodecyl sulfate, $0.137 \mathrm{M} \mathrm{NaCl}, 1 \mathrm{mM} \mathrm{CaCl}, 1$ $\mathrm{mM} \mathrm{MgCl}_{2}, 10 \%$ glycerol, $20 \mathrm{mM}$ Tris [pH 9.0], $0.01 \%$ $\mathrm{NaN}_{3}, 1 \mathrm{mM}$ phenylmethylsulfonyl fluoride). The lysate of
$10^{6}$ cells was incubated with 5 to $10 \mu$ l of serum preadsorbed with an unlabeled protein extract from $10^{7}$ EBV-negative BJA cells. The immune complexes were bound on protein A-Sepharose beads (Pharmacia, Uppsala, Sweden) washed, eluted by boiling in $2 \%$ sodium dodecyl sulfate- $3 \%$ sucrose-5\% mercaptoethanol-20 mM Tris ( $\mathrm{pH}$ 7.0)bromphenol blue, and subjected to polyacrylamide gel electrophoresis as described previously (2). For Western blotting, unlabeled proteins were separated in polyacrylamide gels and electrophoretically transferred onto nitrocellulose (BA85; Schleicher \& Schuell). The nitrocellulose was preincubated with a modified Deinhardt solution (4) for $2 \mathrm{~h}$ and incubated overnight at room temperature with the 1:50 diluted serum. The blots were washed with gelatin buffer $(50$ $\mathrm{mM}$ Tris [pH 7.5], $5 \mathrm{mM}$ EDTA, $150 \mathrm{mM} \mathrm{NaCl}, 0.25 \%$ gelatin, $0.5 \%$ Triton $\mathrm{X}-100,0.1 \%$ sodium dodecyl sulfate) and incubated for another $2 \mathrm{~h}$ with peroxidase-labeled antibodies against human immunoglobulins (Dakopatts, $\mathrm{GmbH}$, Hamburg, Federal Republic of Germany). After subsequently being washed with gelatin buffer, the blot was developed with $0.5 \mathrm{mg}$ of 3,3'-diaminobenzidine per $\mathrm{ml}$ and $0.01 \% \mathrm{H}_{2} \mathrm{O}_{2}-50 \mathrm{mM}$ Tris ( $\mathrm{pH}$ 7.6).

\section{RESULTS}

Fine mapping of the $B$ amHI $\mathrm{Z}$ area with hybrid-selected translation. To analyze the complex protein pattern obtained after hybrid-selected translation with the BamHI Z fragment and P3HR1 RNA (27), we constructed several subclones. Clones pCeZ1622 and pCZR1495 represent the left and the right half of the BamHI Z fragment, respectively, with adjacent sequences. BamHI-e3 (pRe3-521) is the fragment to the left of BamHI-Z and contains mainly sequences from the BZLF2 reading frame. Fragment pCZ453 from the central area of the BamHI Z fragment contains sequences from the BZLF1 reading frame only.

RNA from induced P3HR1 cells, induced B95-8 cells, induced $883 \mathrm{~L}$ cells, and induced Raji cells was used for hybrid selection with the previously mentioned plasmids and, in addition, with the BamHI $\mathrm{R}$ and $\mathrm{Z}$ fragments from strain B95-8 (29). No specific protein above the background could be detected after hybrid-selected translation with the BamHI e3 fragment (Fig. 2). All other fragments tested hybridized with the mRNA for a protein ranging in size from 92 to 96 kilodaltons $(\mathrm{kDa})$ in the different cell lines. With RNA from induced P3HR1 cells, induced Raji cells, and induced B95-8 and 883L cells, 92-, 96-, and 94-kDa proteins, respectively, could be translated in vitro. The size differences were reproducible and were clearly visible in many independent experiments (see Fig. 7). Minor bands in the range of 75 to $83 \mathrm{kDa}$ could be detected in all of these hybrid-selected translations, with a similar size distribution among the different cell lines.

The mRNA for the $80-\mathrm{kDa}$ protein mapped previously in the BamHI R and K fragments (27) hybridized as expected with the BamHI R fragment. However, it did not hybridize to the left segment of the BamHI R fragment contained in the plasmid pCZR1495. Therefore, the protein is encoded by one of the rightward reading frames in the $B a m H I ~ R$ or $K$ fragment, presumably by the BRRF 2 reading frame, according to the sequence data. The protein could only be detected with RNA from induced P3HR1 and particularly from induced B95-8 cells, not from induced $883 \mathrm{~L}$ cells, owing to different responses of the individual cell lines to the induction. In the Raji cells, which could also be induced to a reasonable extent, the protein could not be detected in many 


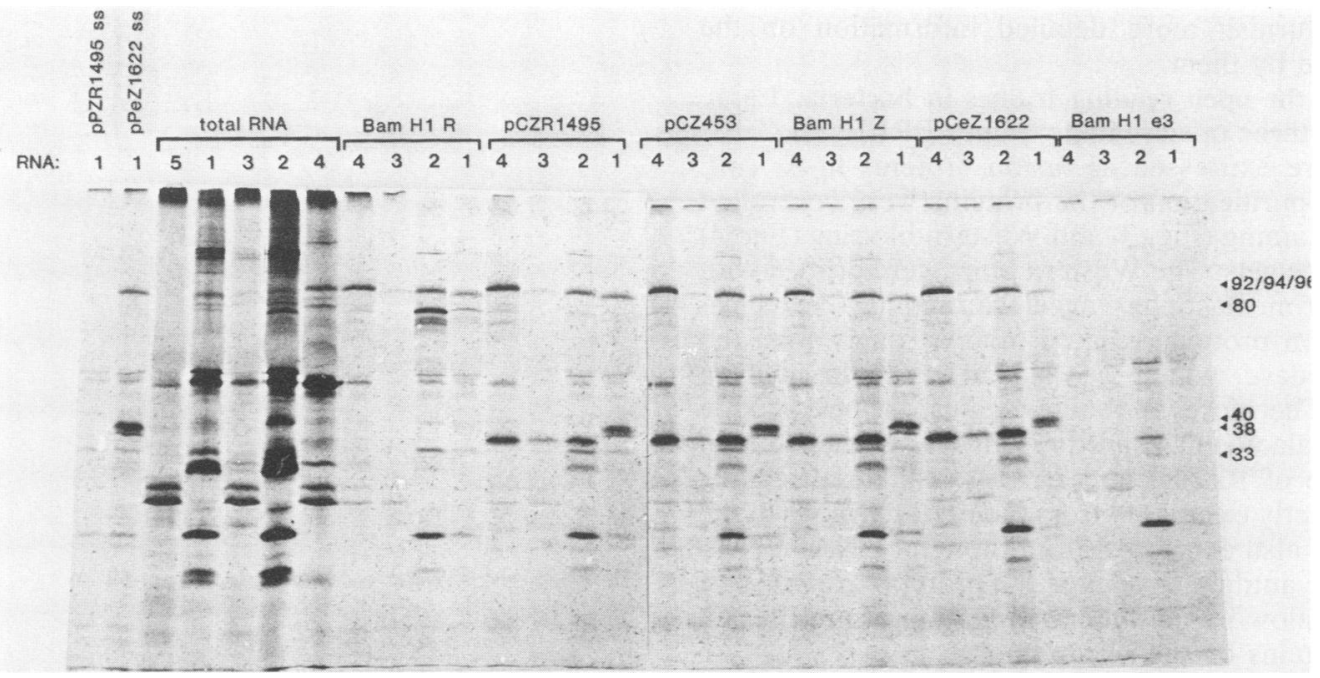

FIG. 2. Hybrid-selected translation with RNAs from induced P3HR1 cells (lanes 1), induced B95-8 cells (lanes 2), induced 883L cells (lanes 3), induced Raji cells (lanes 4), and the DNA fragments indicated. In the left two panels, single-stranded DNA probes from M13 phages representing opposite strands were used. The bound mRNAs and samples of unselected RNA including RNA from EBV-negative BJA cells (lanes 5) as control were translated in vitro with reticulocyte lysate and $\left[{ }^{35} \mathrm{~S}\right]$ methionine as radioactive amino acid. The translation products were immunoprecipitated with a pool of sera from NPC patients and separated on sodium dodecyl sulfate-polyacrylamide gels. The gels were dried, fixed, and exposed to ${ }^{3} \mathrm{H}$-Ultrofilm (LKB Instruments, Bromma, Sweden). Owing to the different numbers of induced cells in the various cell lines, the intensity of the mapped proteins and of the background proteins varied. With RNA from induced B95-8 cells, the most intense signals could be achieved, but the background was also increased owing to overexposure (to make the very thin signals with RNA of induced 883L cells visible). All of the proteins detectable after hybridization with the BamHI e3 fragment represent background proteins. The prominent 23-kDa protein visible after hybridization with all fragments, especially with B95-8 RNA, is encoded by the BLRF1 or the BLRF2 reading frame, or both, in the BamHI L fragment (R. Seibl and H. Wolf, unpublished results).

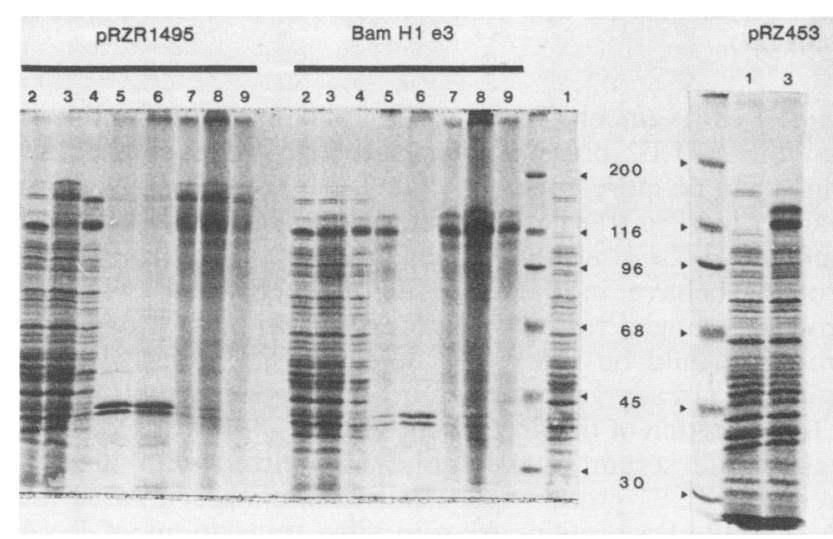

FIG. 3. Coomassie-stained electrophoretically separated bacterial proteins. Bacteria without plasmid (lanes 1), harboring a pUR vector without EBV DNA inserted (lanes 2), or harboring the EBV expression plasmids with the different reading frames fused to the $\beta$-galactosidase reading frame (lanes 3 ) were induced to express the proteins controlled by the lac promoter, lysed, and subjected to electrophoresis. $\beta$-Galactosidase was also included in the marker protein mixture $(116 \mathrm{kDa})$. The expressed $\beta$-galactosidase from the pure pUR plasmid can easily be identified by comparison with the bacteria lacking the plasmid. The fusion proteins were a mixture of proteins translated at full length and proteins in which the EBV segment is present in amounts varying from a few amino acids to nearly full length, as indicated by a smear of proteins above the 116-kDa marker with accumulations at certain preferential molecular masses. The distribution of the different sizes was characteristic for the expressed amino acid sequences. In the purification process, the bacterial lysate was made $3 \%$ with Triton X-100 and the soluble part of the lysate (lanes 4) was discarded. The insoluble part was dissolved in $8 \mathrm{M}$ urea and subsequently dialyzed against $3 \mathrm{M}$ urea. Two bacterial proteins insoluble in $8 \mathrm{M}$ (lanes 5) and $3 \mathrm{M}$ (lanes 6) urea could be separated. The fusion proteins remained dissolved in experiments, indicating that the $80-\mathrm{kDa}$ protein may be a late protein.

From induced Raji, induced B95-8, and induced $883 \mathrm{~L}$ cells, the mRNA for a protein of $38 \mathrm{kDa}$ hybridized to the plasmids pCZR1495, pCZ453, BamHI-Z, and pCeZ1622. Occasionally, the protein was resolved as a double band of 38 and $37.5 \mathrm{kDa}$. With RNA from induced P3HR1 cells, in contrast, a double band of 40 and $39 \mathrm{kDa}$ could be translated after hybridization to the same fragments. To analyze the direction of the transcription, we cloned fragments ZR1495 and eZ1622 into M13mp8 and used single-stranded DNA from phage particles for hybrid-selected translation with P3HR1 RNA. With DNA probes containing both strands of the respective DNA fragments (pCZR1495 and pCeZ1622), the identical pattern of all the proteins encoded by the BamHI Z fragment could be detected. The single-stranded DNA of the M13 clones represents strands of opposite polarity. pPZR1495 single-stranded DNA, which is in the leftward orientation, did not hybridize to any of the translatable mRNAs. With pPeZ1622 single-stranded DNA, a rightward strand, all the proteins could be detected.

In conclusion, the mRNAs for the $92-$ to $96-\mathrm{kD}$ and the 38and $40-\mathrm{kDa}$ proteins both ran in a leftward direction. Only DNA fragments containing sequences from the BZLF1 reading frame hybridized with the transcripts for the 38- and 40-kDa proteins. All fragments containing sequences from the BRLF1 reading frame, as well as fragment pCZ453, hybridized with the transcripts for the $92-$ to $96-\mathrm{kDa}$ proteins. Therefore, it was necessary to prepare monospecific sera against the polypeptides encoded by the different read-

$3 \mathrm{M}$ urea (lanes 7) and could be precipitated by dialysis against phosphate-buffered saline (lanes 8), in which only limited amounts remained in solution (lanes 9 ). 
ing frames to furnish more detailed information on the proteins encoded by them.

Expression of the open reading frames in bacteria. Large segments of the three open reading frames BRLF1, BZLF1, and BZLF2 were expressed as fusion proteins in $E$. coli. Expression and purification of the proteins were controlled by Coomassie staining (Fig. 3) and Western blotting (Fig. 4) of polyacrylamide gels. The Western blots were probed with a serum pool from nasopharyngeal carcinoma (NPC) patients. The fusion proteins reacted more strongly than did pure $\beta$-galactosidase, which was similarly expressed from pUR plasmids. Therefore, antibodies against the polypeptide fused to the $\beta$-galactosidase are present in the sera, in high titer in the case of BZLF2 and BZLF1. All three reading frames are correctly expressed from the pUR plasmids in the bacteria and are also expressed from the viral DNA in NPC patients because antibodies against the proteins are present, as also shown below by immunoprecipitation of the BRLF1 and BZLF1 proteins by the serum pool.

The fusion proteins were purified by a simple and efficient method based upon the relative insolubility of $\beta$ galactosidase fusion proteins. Nearly all the bacterial proteins were soluble in the bacterial lysate with $3 \%$ Triton $X-100$. The mainly insoluble fusion proteins were dissolved in $8 \mathrm{M}$ urea- $0.5 \%$ mercaptoethanol. After dialysis against 3 $M$ urea, another two bacterial proteins could be separated. Most of the still-dissolved fusion proteins precipitated upon dialysis against phosphate-buffered saline, and this sediment was used to immunize rabbits.

Identification of the protein encoded by the BZLF2 reading frame. After in vivo labeling of induced P3HR1, induced B95-8, and superinfected Raji cells, a protein of $140 \mathrm{kDa}$ could be specifically precipitated by the serum of a rabbit immunized with the expressed BZLF2 protein (Fig. 5). As expected, the protein could not be detected in induced Raji cells (data not shown), since the reading frame is deleted in EBV DNA from the Raji strain (22). Therefore, in superinfected Raji cells, the 140-kDa protein was expressed from infecting P3HR1 genomes. No protein could be precipitated from in vitro translations, in accordance with the negative result of the hybrid-selected translations with the

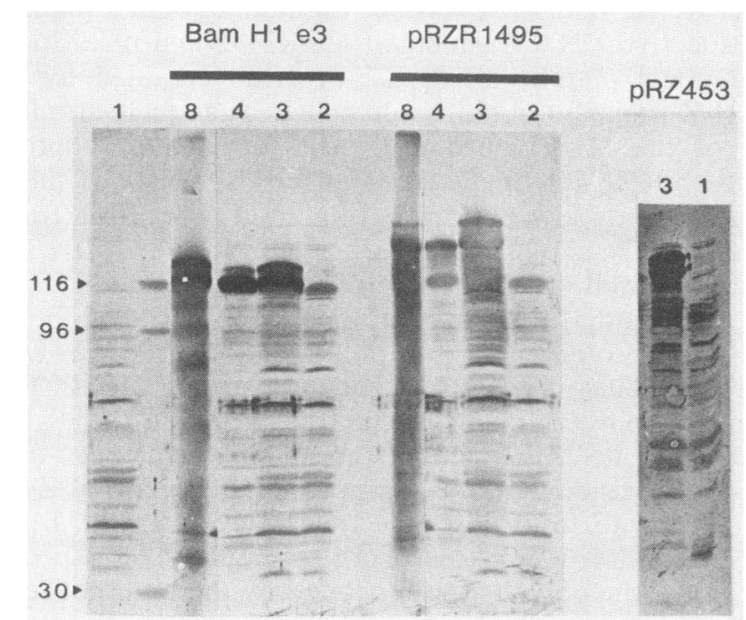

FIG. 4. Western blot of the bacterial lysates and some purification steps described in the legend to Fig. 3. Probes identical to those described in the legend to Fig. 3 were separated in polyacrylamide gels and blotted on nitrocellulose. The blot was immunostained with a pool of sera from NPC patients.

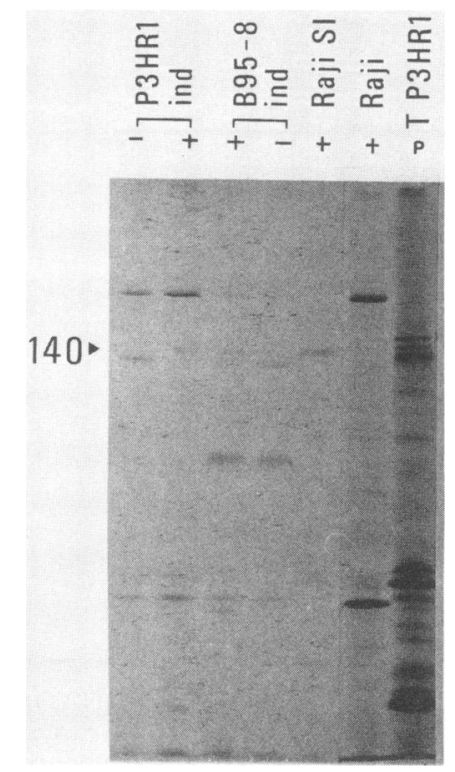

FIG. 5. Immunoprecipitation with rabbit serum against the BZLF2 protein. Induced P3HR1, induced B95-8, superinfected Raji, and Raji cells were labeled in vivo with $\left[{ }^{35}\right.$ S $]$ methionine. The negative serum of the rabbit prior to immunization $(-)$ and the immune serum $(+)$ were used in parallel for immunoprecipitation. An in vitro translation (T) of RNA from induced P3HR1 cells immunoprecipitated with the NPC serum pool (P) was run in parallel as marker. The two prominent proteins in the $140-\mathrm{kDa}$ molecular mass range are the $138-\mathrm{kDa}$ protein encoded by the BALF2 reading frame (17) and the 143-kDa protein encoded by the BNRF1 reading frame (27).

BamHI e3 fragment. The most likely explanation for this is that the BZLF2 protein belongs to the group of proteins which can be more effeciently labeled by in vivo translation than by in vitro translation, which is similar to the situation with the BZLF1 protein. Other proteins, e.g., the BRLF1 protein, behave in the opposite manner. If the BZLF2 protein belonged to the first group, the in vitro translation product would be below the detection limit, because the protein is already very weak in in vivo labeling experiments.

Identification of the protein encoded by the BZLF1 reading frame. The serum of the rabbit immunized with the expressed BZLF1 protein specifically immunoprecipitated the 40- and 39-kDa proteins from in vitro translations of RNA from induced P3HR1 cells and the 38-kDa protein from in vitro translations of RNA from induced B95-8 and induced Raji cells (Fig. 6). From extracts of in vivo-labeled induced B95-8, induced Raji, induced P3HR1, and superinfected Raji cells, a protein of $35 \mathrm{kDa}$ could be immunoprecipitated. Small amounts of the 38- and 40-kDa primary translation products were also detectable. The protein seemed to be posttranslationally cleaved. The additional sequences of the P3HR1 protein seemed to be removed as well. In the superinfected Raji cells, both the 38- and the 40-kDa precursor protein were visible, indicating that the gene might be expressed from infecting P3HR1 as well as endogeneous Raji genomes.

Identification of the protein encoded by the BRLF1 reading frame. By using the serum against the BRLF1 polypeptide, the mapped in vitro-translated proteins of 96,94 , and $92 \mathrm{kDa}$ with RNA from induced Raji cells, induced B95-8 cells, and induced P3HR1 cells, respectively, could be precipitated (Fig. 7). From in vivo-labeled induced B95-8 and induced 


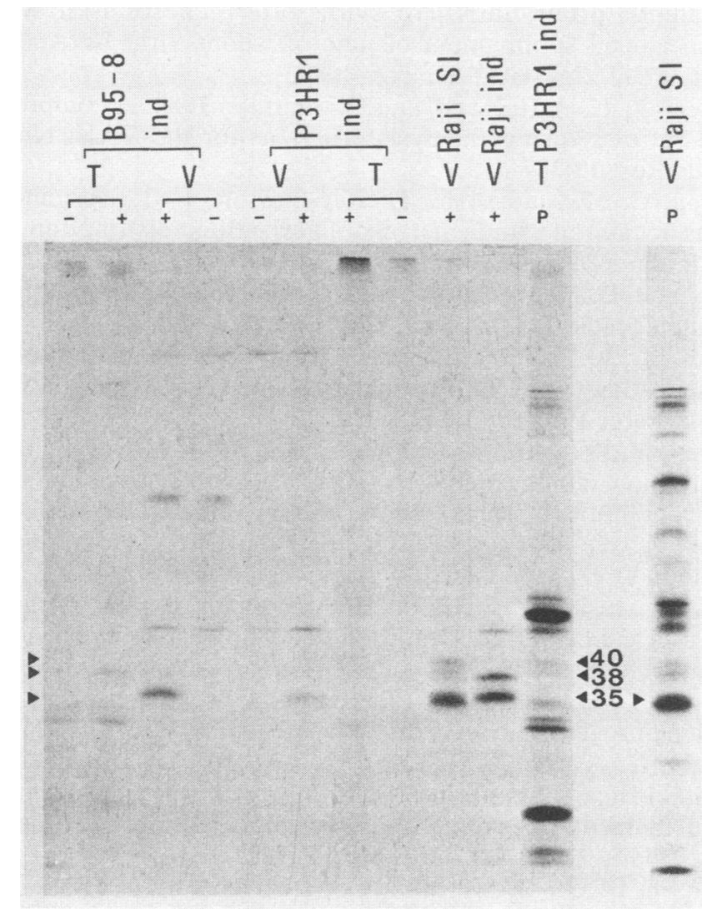

FIG. 6. Immunoprecipitation with the rabbit serum against the BZLF1 protein. The negative serum of the rabbit prior to immunization $(-)$ and the immune serum $(+)$ were used in parallel to immunoprecipitate in vitro translations $(T)$ of RNA from induced B95-8 and induced P3HR1 cells and extracts of in vivo-labeled (V) induced B95-8, induced P3HR1, superinfected Raji, and induced Raji cells. An in vitro translation (T) of unselected RNA from induced P3HR1 cells immunoprecipitated with the NPC serum pool (P) was run in parallel. The serum pool was also used to immunoprecipitate in vivo-labeled superinfected Raji cells, in which the $35-\mathrm{kDa}$ band consists of more than one polypeptide (2). The same is true for the $40-\mathrm{kDa}$ band precipitated by the serum pool from in vitro translations of RNA from induced P3HR1 cells (27).

P3HR1 cells, proteins with molecular masses slightly higher than those of proteins from the corresponding in vitro translation experiments were precipitated. The protein seemed to be posttranslationally modified by the addition of an unknown component. In the induced Raji cells, the protein had the same molecular mass after in vivo or in vitro translation according to sodium dodecyl sulfate-polyacrylamide gel electrophoresis.

\section{DISCUSSION}

We analyzed the transcription and translation of the EBV $B a m H I ~ Z$ area in different cell lines. This region is characterized by considerable strain-specific protein polymorphism. These differences enabled us to demonstrate that in superinfected Raji cells, both the endogeneous Raji genome and the infecting P3HR1 genomes (standard or defective) seem to be expressed in the BamHI Z area.

In contrast to the other cell lines tested, no difference in the expression of B95-8 and 883L cells was observed. B95-8 cells have been established by in vitro transformation of marmoset lymphocytes with virus derived from the $883 \mathrm{~L}$ cell line (15). The expression of the BamHI $\mathrm{Z}$ area remained stable throughout the propagation of the cell lines. This may indicate that the observed protein polymorphism represents strain-specific variations already present in the patients from which the cell lines were established and does not reflect consequences of propagation in cell cultures. Similar strain polymorphism has been reported for herpes simplex virus (20).

The three open reading frames of this area were expressed as fusion proteins with $\beta$-galactosidase in $E$. coli. This approach was chosen because expression of pure eucaryotic proteins in bacteria is, in many cases, not efficient, owing to degradation by proteases or incomplete translation. Even in the $\beta$-galactosidase fusion proteins, the viral segment is frequently not synthesized at full length. A further advantage of the expression as $\beta$-galactosidase fusion protein is the possibility of rapid and efficient purification by successive precipitation. These protein preparations were used to immunize rabbits to generate sera which specifically recognize the protein(s) encoded by the appropriate reading frame.

The reading frame BRLF1 encodes a protein of 93 to 96 $\mathrm{kDa}$, depending on the cell line. Additionally, smaller proteins in the range of 75 to $83 \mathrm{kDa}$ could be mapped to this reading frame. These presumably represent shorter translation products due to truncated mRNAs, which appear in the hybrid-selected translation as indicated by Northern blotting of selected and unselected RNA (data not shown). The BRLF1 protein is posttranslationally modified, at least in induced B95-8 and induced P3HR1 cells, because smaller proteins were precipitated from in vitro translations than from in vivo-labeled cells. The molecular mass calculated from the sequence of the open reading frame is only $67 \mathrm{kDa}$. It cannot be ruled out that additional small coding sequences from elsewhere on the genome, which would not be detected under the stringent hybrid selection conditions or other coding sequences in the BamHI $\mathrm{Z}$ and $\mathrm{R}$ fragments, are fused to BRLF1 by splicing. This seems, however, not very likely, because similar differences in the calculated molecular mass and that obtained by polyacrylamide gel electro-

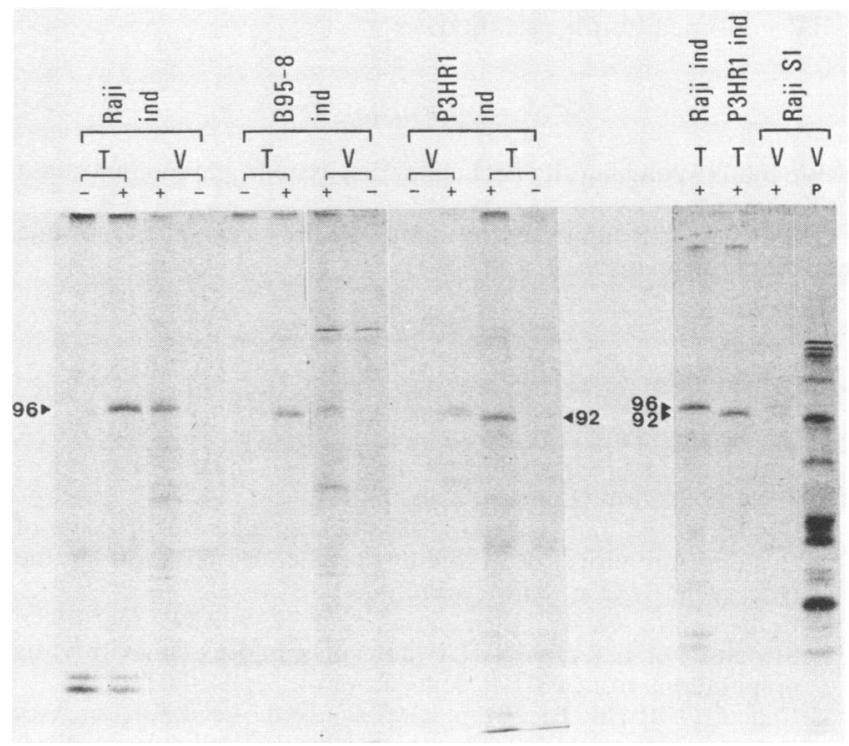

FIG. 7. Immunoprecipitation with the serum against the BRLF1 protein. The negative serum of the rabbit prior to immunization (-) and the immune serum $(+)$ were used in parallel to immunoprecipitate in vitro translations (T) of RNA from induced Raji, induced B95-8, and induced P3HR1 cells and in vivo-labeled extracts (V) of the same cells and of superinfected Raji cells. In vivo-labeled superinfected Raji cells immunoprecipitated with the NPC serum pool $(\mathrm{P})$ were run in parallel. 
phoresis are known from other reading frames. According to our hybridization data, the transcript extends from the BamHI R fragment to the BamHI Z fragment between nucleotides 101750 and 102700 . Sequence analysis has revealed the existence of two TATA boxes near the $5^{\prime}$ end of BRLF1, as well as two suitable downstream polyadenylation sites (1).

The reading frame BZLF1 encodes a $35-\mathrm{kDa}$ protein. The protein is translated into a $38-\mathrm{kDa}$ precursor and seems to be subsequently cloven. In P3HR1 cells, the precursor has a molecular mass of $40 \mathrm{kDa}$, whereas the protein identified by in vivo labeling is of the normal size. In in vitro translation assays, the $35-\mathrm{kDa}$ protein was not detectable, but a second band of $39 \mathrm{kDa}$ in P3HR1 cells and $37.5 \mathrm{kDa}$ in B95-8 and Raji cells, respectively, was seen. The processing may start in the in vitro translation assays but cannot be completed there. The calculated size of the protein, according to the sequence of BZLF1, is only $21 \mathrm{kDa}$. Therefore, it is possible that other reading frames contribute to this protein. The transcript does not hybridize to the neighboring fragments of BamHI-Z. One of the polyadenylation sites discussed for the BRLF1 transcript may be used.

The reading frame BZLF2 encodes a $140-\mathrm{kDa}$ protein; however, the calculated size of the protein translated from BZLF2 is only $25 \mathrm{kDa}$. Therefore, other coding sequences are presumably spliced to this frame. The 5 ' end of the amino acid sequence encoded by BZLF2 consists of a hydrophobic domain which fulfills the criteria of a signal peptide consensus sequence $(21,31,32)$. Signal peptides are responsible for the vectorial translation of secretory and membrane proteins. The $140-\mathrm{kDa}$ EBV membrane protein, reported from several laboratories $(8,18,33)$, may be partially encoded by BZLF2.

With the specific sera against the proteins encoded by the BamHI Z area modification, time-dependent synthesis and cellular localization of the proteins are now accessible for examination, and their role in the trans-induction of latent EBV genomes can be studied.

\section{ACKNOWLEDGMENTS}

We thank Wolfgang Jilg for helpful discussions and immunization of the rabbits.

This work was supported by grant Wo227/4 from the Deutsche Forschungsgemeinschaft.

\section{LITERATURE CITED}

1. Baer, R., A. T. Bankier, M. D. Biggin, P. L. Deininger, P. J. Farrell, T. J. Gibson, G. Hatfull, G. S. Hudson, S. C Satchwell, C. Seguin, P. S. Tuffnell, and B. G. Barrell. 1984. DNA sequence and expression of the B95-8 Epstein-Barr virus genome. Nature (London) 310:207-211.

2. Bayliss, G. J., and $\mathbf{H}$. Wolf. 1981 . The regulated expression of Epstein-Barr virus. III. Proteins specified by EBV during the lytic cycle. J. Gen. Virol. 56:105-118.

3. Cho, M. S., G. W. Bornkamm, and H. zur Hausen. 1984. Structure of defective DNA molecules in Epstein-Barr virus preparations from P3HR1 cells. J. Virol. 51:199-207.

4. Cohen, G. H., B. Dietzschold, M. Ponce de Leon, D. Long, E. Golub, A. Varrichio, L. Pereira, and R. J. Eisenberg. 1984. Localization and synthesis of an antigenic determinant of herpes simplex virus glycoprotein D that stimulates the production of neutralizing antibody. J. Virol. 49:102-108.

5. Cohen, L. K., S. H. Speck, B. E. Roberts, and J. L. Strominger. 1984. Identification and mapping of polypeptides encoded by the P3HR1 strain of Epstein-Barr virus. Proc. Natl. Acad. Sci. USA 81:4183-4187.

6. Countryman, J., and G. Miller. 1985. Activation of expression of latent Epstein-Barr herpesvirus after gene transfer with a small cloned subfragment of heterogeneous viral DNA. Proc. Natl. Acad. Sci. USA 82:4085-4089.

7. Devereux, J., P. Haeberli, and O. Smithies. 1984. A comprehensive set of sequence analysis programs for the VAX. Nucleic Acids Res. 12:387-395.

8. Edson, C. M., and D. A. Thorley-Lawson. 1981. Epstein-Barr virus membrane antigens: characterization, distribution, and strain differences. J. Virol. 39:172-184.

9. Hanahan, D. 1983. Studies on transformation of Escherichia coli with plasmids. J. Mol. Biol. 166:557-580.

10. Luka, J., B. Kallin, and G. Klein. 1979. Induction of the Epstein-Barr virus (EBV) cycle in latently infected cells by N-butyrate. Virology 94:228-231.

11. Masui, Y., T. Mizuno, and M. Inouye. 1984. Novel high-level expression cloning vehicles: $10^{4}$-fold amplification of Escherichia coli minor protein. Biotechnology 2:81-84.

12. Messing, J., R. Crea, and H. Seeburg. 1981. A system for shotgun DNA sequencing. Nucleic Acids Res. 9:309-321.

13. Messing, J., and J. Vieira. 1982. A new pair of M13 vectors for selecting either DNA strand of double-digest restriction fragments. Gene 19:269-276.

14. Miller, G., M. Rabson, and L. Heston. 1984. Epstein-Barr virus with heterogeneous DNA disrupts latency. J. Virol. 50:174-182.

15. Miller, G., T. Shope, H. Lisco, D. Stitt, and M. Lipman. 1972. Epstein-Barr virus: transformation, cytopathic changes, and viral antigens in squirrel monkey and marmoset leukocytes. Proc. Natl. Acad. Sci. USA 69:383-387.

16. Morgan, D. G., G. Miller, J. C. Niederman, H. W. Smith, and J. M. Dowaliby. 1979. Site of Epstein-Barr virus replication in the oropharynx. Lancet i:1154-1157.

17. Motz, M., J. Fan, R. Seibl, W. Jilg, and H. Wolf. 1986. Expression of the Epstein-Barr virus $138-\mathrm{kDa}$ early protein in Escherichia coli for the use as antigen in diagnostic tests. Gene 42:303-312.

18. Müller-Lantzsch, N., B. Georg, N. Yamamoto, and H. zur Hausen. 1980. Epstein-Barr virus-induced proteins. II. Analysis of surface polypeptides from EBV-producing and -superinfected cells by immunoprecipitation. Virology 102:401-411.

19. Norrander, J., T. Kempe, and J. Messing. 1983. Construction of improved M13 vectors using oligodeoxynucleotide-directed mutagenesis. Gene 26:101-106.

20. Pereira, L., E. Cassai, R. W. Honess, B. Roizman, M. Terni, and A. Nahmias. 1976. Variability in the structural polypeptides of herpes simplex virus 1 strains: potential application in molecular epidemiology. Infect. Immun. 13:211-220.

21. Perlman, D., and H. O. Halvorson. 1983. A putative signal peptidase recognition site and sequence in eukaryotic and prokaryotic signal peptides. J. Mol. Biol. 167:391-409.

22. Polack, A., H. Delius, U. Zimber, and G. W. Bornkamm. 1984. Two deletions in the Epstein-Barr virus genome of the Burkitt's lymphoma nonproducer line Raji. Virology 133:146-157.

23. Polack, A., G. Hartl, U. Zimber, U. K. Freese, G. Laux, K. Takaki, B. Hohn, L. Gissmann, and G. W. Bornkamm. 1984. A complete set of overlapping cosmid clones of M-ABA virus derived from nasopharyngeal carcinoma and its similarity to other Epstein-Barr virus isolates. Gene 27:279-288.

24. Rabson, M., L. Heston, and G. Miller. 1983. Identification of a rare Epstein-Barr virus variant that enhances early antigen expression in Raji cells. Proc. Natl. Acad. Sci. USA 80:2762-2766.

25. Rüther, U., and B. Müller-Hill. 1983. Easy identification of cDNA clones. EMBO J. 2:1791-1794.

26. Sample, J., G. Lancz, and M. Nonoyama. 1986. Mapping of genes in BamHI fragment $M$ of Epstein-Barr virus DNA that may determine the fate of viral infection. J. Virol. 57:145-154.

27. Seibl, R., and H. Wolf. 1985. Mapping of Epstein-Barr virus proteins on the genome by translation of hybrid-selected RNA from induced P3HR1 cells and induced Raji cells. Virology 141:1-13.

28. Sixeby, J. W., J. G. Nedrud, N. Raab-Traub, R. A. Hanes, and J. S. Pagano. 1984. Epstein-Barr virus replication in oropharyngeal epithelial cells. N. Engl. J. Med. 310:1225-1230. 
29. Skare, J., and J. L. Strominger. 1980. Cloning and mapping of BamHI endonuclease fragments of DNA from the transforming B95-8 strain of Epstein-Barr virus. Proc. Natl. Acad. Sci. USA 77:3860-3864.

30. Takada, K., N. Shimizu, S. Sakuma, and Y. Ono. 1986. trans activation of the latent Epstein-Barr virus (EBV) genome after transfection of the EBV DNA fragment. J. Virol. 57:10161022.

31. von Heijne, G. 1983. Patterns of amino acids near signalsequence cleavage sites. Eur. J. Biochem. 133:17-21.
32. von Heijne, G., 1985. Signal sequences: the limits of variation. J. Mol. Biol. 184:99-105.

33. Wells, A., N. Koide, and G. Klein. 1982. Two large virion envelope glycoproteins mediate Epstein-Barr virus binding to receptor-positive cells. J. Virol. 41:286-297.

34. Wolf, H., M. Haus, and E. Wilmes. 1984. Persistence of Epstein-Barr virus in the parotid gland. J. Virol. 51:795-798.

35. zur Hausen, H., F. J. O'Neill, and U. K. Freese. 1978. Persisting oncogenic herpesvirus induced by the tumor promoter TPA. Nature (London) 272:373-375. 